\title{
Clinician compliance with laboratory monitoring and prescribing guidelines in HIV 1-infected patients receiving tenofovir
}

\author{
R de Waal, ${ }^{1} \mathrm{MB} \mathrm{ChB}$; K Cohen, ${ }^{2} \mathrm{MB} \mathrm{ChB}, \mathrm{MSc}$, MMed, FCFP (SA); M P Fox, ${ }^{3,4} \mathrm{PhD}$; K Stinson, ${ }^{1,5} \mathrm{PhD}$; \\ G Maartens, ${ }^{2} \mathrm{MB}$ ChB, MMed, FCP (SA); A Boulle, ${ }^{1} \mathrm{MB} \mathrm{ChB}, \mathrm{PhD}$; M-A Davies, ${ }^{1} \mathrm{MB} \mathrm{ChB}, \mathrm{PhD}$ \\ ${ }^{1}$ Centre for Infectious Disease Epidemiology and Research, School of Public Health and Family Medicine, Faculty of Health Sciences, \\ University of Cape Town, South Africa \\ ${ }^{2}$ Division of Clinical Pharmacology, Department of Medicine, Faculty of Health Sciences, University of Cape Town, South Africa \\ ${ }^{3}$ Boston University Center for Global Health and Development, Boston, USA \\ ${ }^{4}$ Health Economics and Epidemiology Research Office, Department of Internal Medicine, School of Clinical Medicine, Faculty of \\ Health Sciences, University of the Witwatersrand, Johannesburg, South Africa \\ ${ }^{5}$ Médecins Sans Frontières, Khayelitsha, Cape Town, South Africa
}

Corresponding author: M-A Davies (mary-ann.davies@uct.ac.za)

Background. Tenofovir is part of the preferred first-line regimen for HIV-infected patients in South Africa (SA), but is associated with kidney toxicity. SA antiretroviral therapy (ART) guidelines recommend creatinine monitoring at baseline (ART start) and at 3, 6 and 12 months, and substituting tenofovir with zidovudine, stavudine or abacavir should creatinine clearance $(\mathrm{CrCl})$ decrease to $<50 \mathrm{~mL} / \mathrm{min}$. Objective. To assess clinician compliance with tenofovir monitoring and prescribing guidelines.

Methods. We described the proportion of adult patients on tenofovir-based first-line ART who were screened for baseline renal impairment, were monitored according to the SA antiretroviral treatment guidelines, and were switched from tenofovir if renal function declined.

Results. We included 13168 patients who started ART from 2010 to 2012. Creatinine concentrations were recorded in 11712 (88.9\%) patients on tenofovir at baseline, $9135 / 11657(78.4 \%)$ at 3 months, $5426 / 10554(51.4 \%)$ at 6 months, and $5949 / 8421(70.6 \%)$ at 12 months. At baseline, 227 (1.9\%) started tenofovir despite a $\mathrm{CrCl}<50 \mathrm{~mL} / \mathrm{min}$. While on tenofovir, 525 patients had at least one $\mathrm{CrCl}$ of $<50 \mathrm{~mL} / \mathrm{min}$. Of 382 patients with $\geq 3$ months' follow-up after a $\mathrm{CrCl}<50 \mathrm{~mL} / \mathrm{min}, 114(29.8 \%)$ stopped tenofovir within 3 months. Clinicians were more likely to stop tenofovir in patients with lower $\mathrm{CrCl}$ and $\mathrm{CD} 4$ count. Of 226 patients who continued to receive tenofovir and had further $\mathrm{CrCls}$ available, $156(69.0 \%)$ had a $\mathrm{CrCl} \geq 50 \mathrm{~mL} / \mathrm{min}$ at their next visit.

Conclusions. Creatinine monitoring is feasible where access to laboratory services is good. Kidney function recovered in most patients who continued to receive tenofovir despite a $\mathrm{CrCl}<50 \mathrm{~mL} / \mathrm{min}$. Further research is needed to determine how best to monitor renal function with tenofovir in resource-limited settings.

S Afr Med J 2016;106(4):369-371. DOI:10.7196/SAMJ.2016.v106i4.10153

Tenofovir, combined with emtricitabine or lamivudine, was introduced to the South African (SA) antiretroviral therapy (ART) guidelines in 2010 as the preferred nucleoside reverse transcriptase inhibitor (NRTI) for HIV-infected patients starting first-line ART. Tenofovir is associated with kidney toxicity, ${ }^{[1]}$ and estimated probabilities of tenofovir discontinuation as a result of kidney toxicity in cohort studies (with durations of follow-up longer than a year) range from $2.3 \%$ to $9.2 \% .^{[2-6]}$ Impairment of kidney function at baseline is an important risk factor for the development of kidney toxicity on tenofovir. ${ }^{[7]}$

While several guidelines recommend excluding kidney disease before starting tenofovir, and monitoring kidney function while on treatment so that tenofovir can be stopped should toxicity develop, some authors argue that tenofovir can be used safely without monitoring. ${ }^{[1,8,9]}$ There is also evidence that clinicians do not always follow monitoring guidelines, even when laboratory monitoring is available. ${ }^{[7,20]}$ The SA 2010 ART guidelines recommended measurement of the serum creatinine concentration before treatment initiation, at 3, 6 and 12 months after initiation and then annually, with calculation of creatinine clearance $(\mathrm{CrCl})$ using the CockcroftGault formula. Tenofovir was contraindicated in patients with a $\mathrm{CrCl}<50 \mathrm{~mL} / \mathrm{min}$. We used retrospective cohort data from two urban SA ART sites to describe clinician compliance with tenofovir prescribing and monitoring guidelines in HIV-infected adults on first-line ART.

\section{Methods}

We included treatment-naive patients aged $\geq 16$ years who started first-line tenofovir-based ART between 2010 and 2012 at Khayelitsha and Themba Lethu clinics in Cape Town and Johannesburg, SA, respectively. Both are public sector HIV clinics that manage patients according to standard national ART protocols. ${ }^{[11,12]}$

We calculated the proportions of patients who had creatinine concentration recorded at baseline (between 6 months before and 2 weeks after treatment initiation) and at 3 months (0.5 - 4 months), 6 months (4 - 8 months) and 12 months (8 - 18 months) after treatment initiation. We calculated $\mathrm{CrCl}$ using the Cockcroft-Gault formula: $\mathrm{C}_{\mathrm{Cr}}(\mathrm{mL} / \mathrm{min})=[(140-$ age $) \times$ weight $(\mathrm{kg})](\times 0.85$ if female $) / \mathrm{S}_{\mathrm{Cr}}$ $(\mu \mathrm{mol} / \mathrm{L}){ }^{[13]}$ Patient weights were not always available on the same date as creatinine concentrations. For baseline $\mathrm{CrCls}$ we matched the closest weight to each creatinine concentration if both were within the baseline window, and they were within 30 days of each other. From 2 weeks to 6 months after treatment initiation we matched the closest weight to each creatinine concentration if they were within 
14 days of each other. After 6 months we carried weights forward for up to 9 months if weights were missing. We calculated the proportion of patients who stopped tenofovir within 3 months of a $\mathrm{CrCl}<50 \mathrm{~mL} /$ min, either at baseline or while on treatment (excluding those who switched to second-line regimens because of virological failure). We defined time on tenofovir as the time from treatment initiation until stopping tenofovir or the end of follow-up. Multivariate logistic regression analysis was used to identify factors associated with stopping tenofovir in patients who had a $\mathrm{CrCl}<50 \mathrm{~mL} / \mathrm{min}$ while on treatment, and Stata 13.0 was used for the statistical analyses.

The University of Cape Town Faculty of Health Sciences Human Research Ethics Committee approved the study (576/2011). The research ethics committees of the universities of Cape Town and the Witwatersrand approved the sites' contribution of data to the study.

\section{Results}

We included 13168 patients on tenofovir-based first-line ART. Their baseline characteristics are shown in Table 1 . The most frequently prescribed concomitant antiretrovirals were lamivudine (13 003 patients) and efavirenz (11 641 patients).

Creatinine concentrations were recorded in 11712 (88.9\%) patients at baseline, in $9135 / 11657$ (78.4\%) who were on tenofovir at 3 months, in $5426 / 10554$ (51.4\%) at 6 months, and in $5949 / 8421$ (70.6\%) at 12 months. Creatinine concentrations were recorded at both baseline and 3 months in 8406 patients (71.8\%) but at all four time points in only 1742 (20.7\%) of those followed up for 12 months. We were able to calculate $\mathrm{CrCl}$ for $79.4 \%$ of visits where creatinine concentrations were recorded in the database.

Two hundred and twenty-seven (2.2\%) of the 10416 patients who had creatinine concentrations recorded at baseline started tenofovir despite a $\mathrm{CrCl}<50 \mathrm{~mL} / \mathrm{min}$. Of these 227 patients, 185 had at least 3 months' follow-up after starting tenofovir, and tenofovir was stopped (within 3 months) in 26 (14.1\%). In 119 (81.5\%) of the 146 patients who continued to receive tenofovir and had further creatinine concentrations available, the $\mathrm{CrCl}$ was $\geq 50 \mathrm{~mL} / \mathrm{min}$ at their next visit. Among the patients with a $\mathrm{CrCl}<50 \mathrm{~mL} / \mathrm{min}$, the $\mathrm{CrCl}$ was $\geq 50 \mathrm{~mL} /$ min at their following visit in 5 and was stable at their following visit in 16, while continuing to receive tenofovir. In 2 patients, $\mathrm{CrCl}$ deteriorated at their following visit: one stopped tenofovir and the $\mathrm{CrCl}$ improved to the baseline level, and the other continued to receive tenofovir and the $\mathrm{CrCl}$ subsequently improved to the baseline level. Four patients had no further creatinine concentrations on tenofovir available.

A further 525 patients had at least one $\mathrm{CrCl}<50 \mathrm{~mL} / \mathrm{min}$ while on treatment. Tenofovir was stopped in $114(29.8 \%)$ of the 382 patients with at least 3 months' follow up after the low $\mathrm{CrCl}$ result. In $156(69.0 \%)$ of the 226 patients who continued to receive tenofovir and had further creatinine concentrations available, the $\mathrm{CrCl}$ was $\geq 50 \mathrm{~mL} / \mathrm{min}$ at their next visit. In the patients with a $\mathrm{CrCl}<50 \mathrm{~mL} /$ $\mathrm{min}$, the $\mathrm{CrCl}$ was $\geq 50 \mathrm{~mL} / \mathrm{min}$ at their following visit in 21 and was $<50 \mathrm{~mL} / \mathrm{min}$ but stable or improving at their following visit in 37, while continuing to receive tenofovir. In 2 patients, $\mathrm{CrCl}$ deteriorated at their following visit: both continued to receive tenofovir and $\mathrm{CrCl}$ subsequently improved. Ten patients had no further creatinine concentrations on tenofovir available.

Logistic regression analysis, adjusting for treatment site, in the 525 patients who had a $\mathrm{CrCl}<50 \mathrm{~mL} / \mathrm{min}$ while on treatment showed that clinicians were more likely to stop tenofovir in patients with a lower $\mathrm{CrCl}$ (odds ratio (OR) 1.14; 95\% confidence interval (CI) $1.02-1.28$ for every $5 \mathrm{~mL} / \mathrm{min}$ decrease in $\mathrm{CrCl}$ ), and were also more likely to stop in patients with lower CD4 cell counts (OR 1.13; 95\% CI 1.00 - 1.29 for every 50 cells/ $\mu \mathrm{L}$ decrease in CD4 cell count) (Table 2).
Table 1. Patient baseline characteristics

\begin{tabular}{ll}
\hline Total patients, $N$ & 13168 \\
Males, $n(\%)$ & $4923(37.4 \%)$ \\
Age (years), median (IQR) & $35.5(30.0-42.1)$ \\
CD4 count (cells/ $\mu \mathrm{L})$, median (IQR) ${ }^{*}$ & $166(83-239)$ \\
Year of ART initiation, $n(\%)$ & \\
$\quad 2010$ & $3405(25.8)$ \\
2011 & $4985(37.9)$ \\
2012 & $4778(36.3)$ \\
Follow-up time on tenofovir (months), median (IQR) & $14.5(6.8-24.1)$ \\
IQR = interquartile range. &
\end{tabular}

Table 2. Associations with stopping tenofovir after $\mathrm{CrCl}<50 \mathrm{~mL} / \mathrm{min}$

\begin{tabular}{|c|c|c|c|c|c|}
\hline \multirow[b]{2}{*}{ Risk factor } & \multirow[b]{2}{*}{$n$} & \multicolumn{2}{|c|}{ Unadjusted } & \multicolumn{2}{|c|}{ Adjusted $^{\star}$} \\
\hline & & OR & $95 \%$ CI & OR & 95\% CI \\
\hline $\begin{array}{l}\mathrm{CrCl}(/ 5 \mathrm{~mL} / \mathrm{min} \\
\text { decrease })\end{array}$ & 525 & 1.03 & $0.95-1.12$ & 1.14 & $1.02-1.28$ \\
\hline $\begin{array}{l}\text { CD } 4 \text { count } \\
\text { (/50 cells } / \mu \mathrm{L} \text { decrease })\end{array}$ & 388 & 1.22 & $1.09-1.37$ & 1.13 & $1.00-1.29$ \\
\hline
\end{tabular}

There were no significant associations between sex, age, concomitant antiretrovirals or viral load and stopping tenofovir.

\section{Discussion}

We found that clinician compliance with monitoring guidelines was good at baseline and reasonable thereafter. Tenofovir was stopped only in a minority of patients whose $\mathrm{CrCl}$ deteriorated to $<50 \mathrm{~mL} /$ min after starting tenofovir. However, $\mathrm{CrCl}$ recovered to $\geq 50 \mathrm{~mL} /$ min in most patients who did not stop tenofovir. Similarly, $\mathrm{CrCl}$ recovered in most patients who started tenofovir despite a baseline $\mathrm{CrCl}$ of $<50 \mathrm{~mL} / \mathrm{min}$.

Data regarding clinician compliance with tenofovir creatinine monitoring guidelines are limited. Our findings compare favourably with those of Chua et al. ${ }^{[10]}$ who conducted a retrospective cohort analysis in $226 \mathrm{HIV}$-infected patients in Singapore and found that $73.5 \%$ of patients had creatinine measured at baseline, $84.9 \%$ within the first 6 months of treatment, $53 \%$ at 12 months and $77 \%$ at 24 months. Eighteen patients had a $\mathrm{CrCl}<50 \mathrm{~mL} / \mathrm{min}$ after tenofovir initiation and tenofovir was stopped because of kidney toxicity in 2 (11\%). In 10 patients $\mathrm{CrCl}$ improved despite continuing tenofovir without dose adjustment.

\section{Study limitations}

Our study has several limitations. We analysed data collected for routine clinical practice, and there were missing data. Both cohort databases were linked to electronic laboratory records to obtain as many creatinine records as possible. However, we may have underestimated clinician compliance with monitoring guidelines if creatinine was measured but results were not recorded in the database. Also, results in the database may not have been communicated to clinicians because of clerical errors, so we were not able to determine whether or not clinicians who did not stop tenofovir in patients with a low $\mathrm{CrCl}$ had actually received the 
abnormal results. Weight data relied solely on manual data entry, and there was a high proportion of missing weights. The cohort database does not routinely capture adverse drug reaction data, and many reasons for stopping drugs were missing, so we cannot ascertain accurately whether tenofovir stops were in fact due to kidney toxicity.

\section{Conclusions}

Our study demonstrates that good compliance with creatinine monitoring guidelines is feasible in SA public sector settings with reasonable access to laboratory services. The large proportion of patients whose renal function improved despite continuing tenofovir is reassuring for settings with poor access to laboratory monitoring. However, clinicians substituted tenofovir with an alternative NRTI in patients with more severe renal impairment and immunosuppression. We therefore cannot conclude that it is safe to prescribe tenofovir without any creatinine monitoring. Further research is needed to inform guidelines regarding how and when best to monitor patients on tenofovir, particularly in resource-limited settings.

Acknowledgement. This research was supported by the President's Emergency Plan for AIDS Relief (PEPFAR) through the Centers for Disease Control and Prevention (CDC) under the terms of Cooperative Agreement Number GH000371. Its contents are solely the responsibility of the authors and do not necessarily represent the official views of the CDC.

\section{References}

1. Cooper RD, Wiebe N, Smith N, et al. Systematic review and meta-analysis: Renal safety of tenofovir disoproxil fumarate in HIV-infected patients. Clin Infect Dis 2010;51(5):496-505. DOI:10.1086/65568 2. Madeddu G, Bonfanti P, de Socio GV, et al. Tenofovir renal safety in HIV-infected patients: Results from the SCOLTA Project. Biomed Pharmacother 2008;62(1):6-11. DOI:10.1016/j.biopha.2007.04.008

3. Nishijima T, Komatsu H, Gatanaga H, et al. Impact of small body weight on tenofovir-associated renal dysfunction in HIV-infected patients: A retrospective cohort study of Japanese patients. PLoS One 2011;6(7):e22661. DOI:10.1371/journal.pone.0022661

4. Patel KK, Patel AK, Ranjan RR, et al. Tenofovir-associated renal dysfunction in clinical practice:

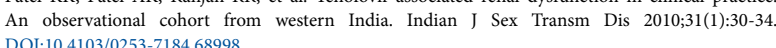

5. Antoniou T, Raboud JM, Chirhin S, et al. Incidence of and risk factors for tenofovir-induced nephrotoxicity: A retrospective cohort study. HIV Med 2005;6(4):284-290. DOI:10.1111/j.14681293.2005.00308.x

6. Calza L, Trapani F, Tedeschi S, et al. Tenofovir-induced renal toxicity in 324 HIV-infected, antiretroviral-naive patients. Scand J Infect Dis 2011;43(8):656-660. DOI:10.3109/00365548.2011.57 2906

7. Brennan A, Evans D, Maskew M, et al. Relationship between renal dysfunction, nephrotoxicity and death among HIV adults on tenofovir. AIDS 2011;25(13):1603-1609. DOI:10.1097/ QAD.0b013e32834957da

8. DART trial team. Routine versus clinically driven laboratory monitoring of HIV antiretroviral therapy in Africa (DART): A randomised non-inferiority trial. Lancet 2010;375(9709):123-131. DOI:10.1016 S0140-6736(09)62067-5

9. Johnson DC, Chasela C, Maliwichi M, et al. Tenofovir use and renal insufficiency among pregnant and general adult population of HIV-infected, ART-naive individuals in Lilongwe, Malawi. PLoS One 2012;7(7):e41011. DOI:10.1371/journal.pone.0041011

10. Chua AC, Llorin RM, Lai K, et al. Renal safety of tenofovir containing antiretroviral regimen in a Singapore cohort. AIDS Res Ther 2012;9(19):1-5. DOI:10.1186/1742-6405-9-19

11. Boulle A, van Cutsem G, Hilderbrand $K$, et al. Seven-year experience of a primary care antiretroviral treatment programme in Khayelitsha, South Africa. AIDS 2010;24(4):563-572. DOI:10.1097 QAD.0b013e328333bfb7

12. Fox MP, Maskew M, MacPhail AP, et al. Cohort profile: The Themba Lethu Clinical Cohort, Johannesburg, South Africa. Int J Epidemiol 2013;42(2):430-439. DOI:10.1093/ije/dys029

13. National Kidney Foundation. K/DOQI Clinical Practice Guidelines for Chronic Kidney Disease Evaluation, Classification and Stratification. Am J Kidney Dis 2002;39(2 Suppl 1):S1-S266 DOI:10.1053/ajkd.2002.30940

Accepted 14 October 2015. 